\title{
Effectiveness and Safety of Polydioxanone Thread Embedding Acupuncture Compared to Physical Therapy in the Treatment of Patients with Non- Specific Chronic Neck Pain: An Assessor-Blinded, Randomized, Controlled, Clinical Trial
}

This article was published in the following Dove Press journal: Journal of Pain Research

Jae Ik Kim, $\mathbb{D}^{1, *}$ ChangHyun Han, (iD) $2,3, *$ Ju Hyun Jeon, ' Jin Youp Kim, (10 ${ }^{4}$ Ojin Kwon, (D) ${ }^{2}$ So-Young Jung, ${ }^{2}$ Jun-Hwan Lee, $\mathbb{D}^{2,3}$ Changsop Yang, $\mathbb{D}^{2}$ Eunseok Kim, 'Y Young II Kim (D)'

'Department of Acupuncture \& Moxibustion Medicine, College of Korean Medicine, Daejeon University, Daejeon, Republic of Korea; ${ }^{2}$ Clinical Medicine Division, Korea Institute of Oriental Medicine, Daejeon, Republic of Korea; ${ }^{3}$ Korean Medicine Life Science, University of Science \& Technology (UST), Campus of Korea Institute of Oriental Medicine, Daejeon, Republic of Korea; ${ }^{4}$ Department of Clinical Korean Medicine, Graduate School, Kyung Hee University, Seoul, Republic of Korea

*These authors contributed equally to this work

Correspondence: Young II Kim Department of Acupuncture \& Moxibustion Medicine, Daejeon University Daejeon Korean Medicine Hospital, 75, Daedeokdaero 176, Seo-gu, Daejeon, Republic of Korea

Tel +82-42-470-9137

Fax +82-42-477-9005

Email omdkim0I@dju.kr

Eunseok Kim

Department of Acupuncture \& Moxibustion Medicine, Daejeon University Seoul Korean Medicine Hospital, 32, Beobwon-ro II, Songpa-gu, Seoul, Republic of Korea

Tel +82-2-2222-8I03

Fax +82-2-2222-8III

Email mybestcando@naver.com
Background: Thread embedding acupuncture (TEA) has recently been used as a conservative treatment method for non-specific chronic neck pain (CNP). The objective of this study was to evaluate the effectiveness and safety of TEA compared to physical therapy (PT) for treating patients with CNP.

Methods: A total of 128 patients diagnosed with CNP were randomly assigned to a TEA group and a PT group at a 1:1 ratio. The TEA group received four sessions of TEA, while the PT group received eight sessions of PT over 4 weeks. Outcomes were assessed using Neck Pain and Disability Scale (NPDS), clinically important difference (CID), cervical spinal angle, Beck Depression Inventory II (BDI-II), Beck Anxiety Inventory (BAI), Patient Global Impression of Change (PGIC), and EuroQol Five-Dimension (EQ-5D) at baseline and 5, 9, and 13 weeks.

Results: The TEA group showed significant improvement in NPDS compared to the PT group at 5, 9, and 13 weeks. Proportions of patients with decreased NPDS scores of more than 11.5 points (minimal CID) were significantly higher in the TEA group at 5, 9, and 13 weeks. There were significant differences between the two groups at 5, 9, and 13 weeks for BDI-II, and at 5 and 9 weeks for BAI. For EQ-5D, the TEA group showed significant improvement at 5, 9, and 13 weeks. There was no significant difference in cervical spinal angle between the two groups. For PGIC, better improvement was observed at 9 and 13 weeks in the TEA group. Adverse events associated with interventions were mostly temporary and mild.

Conclusion: For patients with CNP, TEA treatment was found to be more effective than PT treatment for improving their pain and dysfunction, quality of life, and psychological distress. Despite some post-treatment discomfort, TEA treatment can be considered as a useful treatment method for patients with CNP.

Trial Registration: This trial has been registered 5 April 2019 in Clinical Research Information Service of South Korea (CRIS- KCT0003720).

Keywords: thread embedding acupuncture, physical therapy, non-specific chronic neck pain, randomized controlled trial

\section{Background}

Non-specific chronic neck pain (CNP) is neck pain that lasts for more than 3 months without neurological abnormalities, structural pathology, or traumatic underlying causes. CNP is classified as cervicalgia (M54.2), cervical spondylosis 
without myelopathy or radiculopathy (M47.8), Myalgia, other (M79.18), etc., based on the international classification of diseases-10. ${ }^{1} \mathrm{CNP}$ is one of the most common musculoskeletal disorders, and is prevalent in about $26 \%$ to $71 \%$ of the adult population. ${ }^{2,3}$ It recurs frequently, alternating between aggravation and improvement, and is likely to persist as a chronic condition, resulting in decreased quality of life and increased health care cost. ${ }^{4,5}$

Acupuncture is widely used in patients with musculoskeletal pain, and several studies on the acupuncture treatment of neck pain have been conducted. ${ }^{6-8}$ Thread embedding acupuncture (TEA) is a treatment that transmits peripheral stimuli to central nerves by embedding a thread in acupuncture points and reforms soft tissues at the treatment site. ${ }^{9}$ TEA has been widely used in recent years, and its continuous stimulating effect is considered to allow long-term effects to be maintained with relatively few treatments. ${ }^{10}$ Recently, many studies investigated TEA using catgut in other countries, ${ }^{11-13}$ while, in the Republic of Korea, polydioxanone (PDO) TEA is mainly used. PDO is a synthetic absorbable suture, which is absorbed completely within 180 days via hydrolytic degradation, whereas catgut is digested by proteolytic enzymes in the body. ${ }^{14}$ Clinical studies on PDO TEA for treating patients with CNP have also been carried out. One study applied PDO TEA and Sham TEA to patients with CNP, ${ }^{15}$ while another evaluated the add-on effect of TEA treatment to usual care in the treatment of patients with CNP. ${ }^{16}$

Physical therapy (PT) is widely used in the treatment of patients with chronic musculoskeletal pain, including CNP. ${ }^{17-20}$ In particular, in the Republic of Korea, according to one research survey, heat therapy was the most commonly used treatment, followed by interfering wave therapy. ${ }^{21}$ Although a previous study that evaluated the add-on effect of TEA treatment to usual care including PT has been conducted, ${ }^{16}$ treatments other than PT were also allowed for the control group. To our knowledge, no previous studies have compared the effectiveness and safety of TEA to those of PT in the treatment of patients with CNP. Although PT and TEA were inherently different treatment methods, comparing the effects of TEA with PT, which is generally prescribed, was expected to provide meaningful clinical insight and evidence. Therefore, we performed an assessor-blinded, randomized controlled, clinical trial to evaluate the effectiveness and safety of PDO TEA compared to PT, in the treatment of patients with CNP.

\section{Methods}

\section{Study Design and Participants}

A total of 128 patients diagnosed with CNP were recruited in this study. They visited Daejeon University Dunsan Korean Medicine Hospital from April 08, 2019 to December 20, 2019. This clinical trial was performed as a 2 -arm parallel design, assessor-blinded, randomized controlled, stratified block $^{22-27}$ (male, female). Participants were randomly assigned in a 1:1 ratio to either the TEA or PT group. The TEA group received a total of 4 TEA treatments, while the PT group received a total of 8 PT treatments over 4 weeks. This clinical trial was approved by the Institutional Review Board (DJDSKH-19-BM-04), registered with the Clinical Research Information Service (KCT0003720), and conducted in accordance with the Declaration of Helsinki.

We assumed that the mean difference between the changes in Neck Pain and Disability Scale (NPDS) before and after treatment in each group was 11.5 , and the standard deviation was 20.67 , based on the clinical significance and a previous study. ${ }^{16,28}$ A significance level of $5 \%$ and a power of $80 \%$ were applied. As a result, 51 patients were needed for each group, and considering the dropout rate of $20 \%$, a total of 128 patients (64 per group) were recruited. The detailed process of calculating sample size is described in the protocol paper. ${ }^{29}$ All applicants were provided a written explanation and an informed consent form, and received a full explanation of this trial.

Eligibility criteria were as follows: ${ }^{14-16,29-33}$ A subject 1) is aged (19-65) years-old, and 2) have suffered from neck pain for over three months, ${ }^{24} 3$ ) scores more than $40 \mathrm{~mm}$ on the visual analogue scale (VAS) at the time of screening, 4) is diagnosed with CNP by clinicians based on image examination, history taking, physical and medical examination, and 5) is willing and able to observe the intervention and follow-up assessments. The exclusion criteria were: An excluded subject 1) suffers radicular pain in the upper limb with the distribution of a particular nerve root, 2) demonstrates neurological abnormality, such as positive to special test, such as Bakody sign (shoulder abduction relief sign test), Spurling test, muscle atrophy, paraesthesiae (sensory deficits), hyporeflexia of deep tendon reflex, weakness, pathologic reflex, 3) demonstrates major spinal pathology, such as spondylitis, myelopathy, fracture, neoplasm, neuromyopathic scoliosis, congenital abnormality, 4) shows a history of spinal surgery or scheduled procedures during the study, 5) suffers from pain more severe than neck pain in another region, 6) suffers from uncontrolled low back pain, ${ }^{31-33} 7$ ) shows a history of receiving 
TEA at the neck region in the past 6 months, ${ }^{14} 8$ ) suffers from skin damage that is difficult to treat on the neck, 9) has shown hypersensitive reaction to previous acupuncture treatment, metal allergy, severe atopy, keloid, or other skin hypersensitivities, 10) suffers from hemorrhagic disease or factors that can affect hemostasis, such as the taking of antiplatelet drug or anti-coagulant, 11) is pregnant, or is planning pregnancy during the trial, 12) suffers from cardiovascular disease or uncontrolled diabetes, 13) shows history of major psychiatric or neurotic disability or cognitive instability, 14) shows history of alcoholism or drug abuse, 15) suffers from severe underlying disease requiring active therapy, or 16) was considered by the researcher to be inappropriate for this trial.

\section{Randomization and Blinding}

Randomization was conducted so as not to be biased, and was blinded to researchers and subjects. Statistician who was not involved in this study randomly assigned 64 patients to each group. A gender-based stratified randomization was performed based on previous studies demonstrating relatively improved response to pain and quality of life in women compared to men and higher preference in Korean Medicine. ${ }^{2-27}$ The generated randomization table and the file were protected from disclosure. The detailed randomization procedure is described in the protocol paper. ${ }^{29}$

To reduce bias as much as possible, this trial was designed to be assessor-blinded. The assessor did not perform the intervention, did not know what kind of treatment the subject received, and asked only simple questions about the contents and evaluation items to fill out the case report form (CRF). However, due to the characteristics of the procedure, blinding of the subject and practitioner to the intervention was impossible.

\section{Interventions}

In the TEA group, a total of 4 TEA treatments were administered once a week for 4 weeks. The TEA of 29 gauge in diameter and 30 or $40 \mathrm{~mm}$ in length (29X36DF, 29046DF, Dongbang Medical CO., Seongnam, Republic of Korea) was selectively used, considering the muscle condition of the subject, and the depth of penetration. The practitioner inserted the TEA, and immediately withdrew it, without further stimulation (Figure 1). SI12, SI14, BL10, BL11, BL12, TE15, TE16, LI17, GB20, GB21, and EX-B2 were selected as acupoints pool. The practitioner selected 4 to 8 insertion points per side from acupoints pool, according to the subject's symptom. On the
A

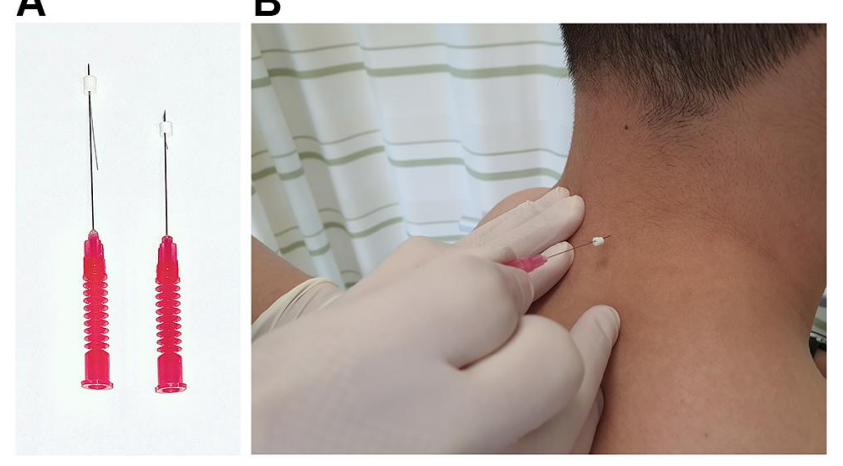

Figure I Application of polydioxanone thread embedding acupuncture. (A) 29 gauge $\times 40 \mathrm{~mm}$ needle (left) and 29 gauge $\times 30 \mathrm{~mm}$ needle (right). (B) needle insertion to EX-B2.

paravertebral point of dorsal aspect, an oblique or perpendicular insertion was performed; and on the lateral side of the cervical vertebrae and thorax, a transverse or oblique insertion was performed, to avoid damaging critical structures. Before and after the treatment, cotton saturated with $78 \%$ alcohol was used to sterilize the neck region.

In the PT group, a total of 8 PT treatments were administered twice a week for 4 weeks. The PT group received heat therapy using hot pack (DS-3860H, Daeshinelc Co., Bucheon, Republic of Korea) and interferential current therapy (ICT) (EDiT 400, Nemectron, Karlsruhe, Germany). The ICT pads were attached to four points of the neck pain area, and hot pack was applied to the neck region at the same time for about 15 minutes per session. ${ }^{34}$

The subject in both groups received rescue medication (Acetaminophen $500 \mathrm{mg}$, up to six tablets per day). Detailed information of the intervention and cointerventions is described in the protocol paper. ${ }^{29}$

\section{Outcome Measures}

Assessments were conducted at baseline, and 5, 9, and 13 weeks $(5 \mathrm{~W}, 9 \mathrm{~W}$, and $13 \mathrm{~W}$, respectively). The treatment period was from baseline to 4 weeks. The primary outcome was the mean changes in the NPDS score at 9W (8 weeks after randomization). ${ }^{6,35,36}$ The secondary outcomes included NPDS scores at $5 \mathrm{~W}$ and $13 \mathrm{~W}$, clinically important difference (CID), ${ }^{28,37,38}$ cervical spinal angle, ${ }^{39-45}$ Beck Depression Inventory II (BDI-II), ${ }^{46-48}$ Beck Anxiety Inventory (BAI), ${ }^{46,49,50}$ EuroQol Fivedimensions 3-level version (EQ-5D-3L), ${ }^{51,52}$ Patient Global Impression of change (PGIC). ${ }^{53}$

The researcher assessed the adverse events (AEs) at every visit based on vital signs, examinations, and history taking. 
The severity of AEs related to intervention, and the causal relationships between intervention and AEs were evaluated. ${ }^{54,55}$ Also, we classified local discomforts that lasted more than 2 days after TEA treatment into AEs from (2 to 7) days (delayed AEs) and AEs for more than 7 days, based on the results of previous study in which some subjects complained of local discomfort for a few days. ${ }^{56}$

\section{Statistical Analysis}

The statistical program SAS version 9.4 (SAS Institute Inc., Car, NC) was used, and when missing values were encountered, multiple imputations were used. The data were analyzed by Full Analysis Set (FAS) and Per Protocol (PP) set. Fisher Exact test or independent $t$ test was used to verify the homogeneity between the two groups at baseline. To compare the mean changes in the NPDS, BDI-II, BAI, EQ-5D, Analysis of covariance (ANCOVA) was used with score at baseline as a covariate. Within each group, to compare the outcomes before and after treatment, the paired $t$-test was used. To analyze changes over time, Repeated measures analysis of variance (RM-ANOVA) was performed.

\section{Results}

Between April 08, 2019 and December 20, 2019, a total of 138 participants were screened for eligibility. Of these 138, 10 did not meet the inclusion criteria, or withdrew consent. Among the 128 eligible patients, 116 completed the study, while 12 dropped out ( 8 in the TEA group, 4 in the PT group) (Figure 2). The 12

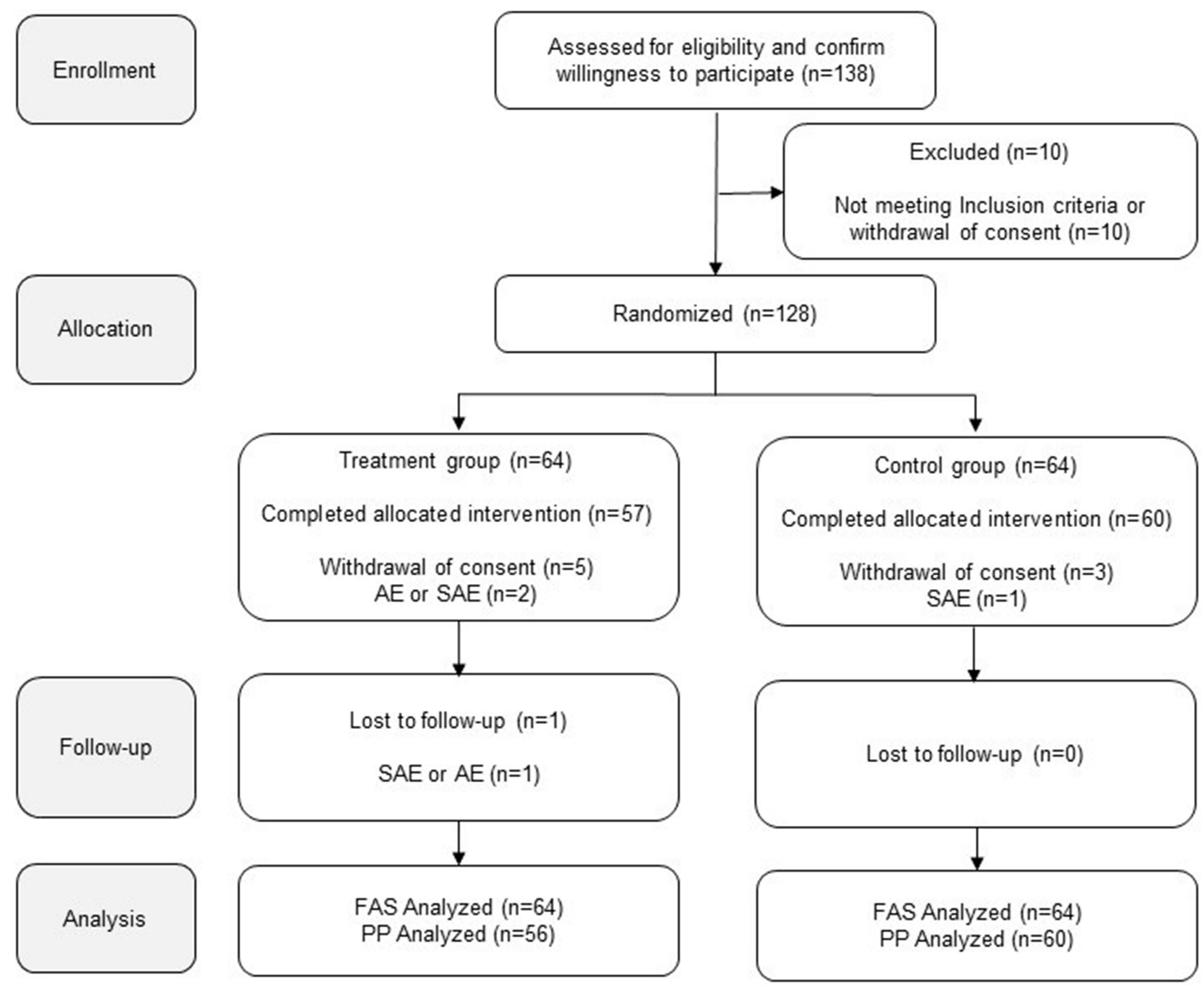

Figure 2 Study flow.

Abbreviations: AE, adverse event; FAS, full analysis set; PP, per protocol; SAE, severe adverse event. 
Table I Demographics and Clinical Characteristics at Baseline

\begin{tabular}{|c|c|c|c|c|}
\hline \multicolumn{2}{|c|}{ Characteristics } & \multirow{2}{*}{$\begin{array}{l}\text { TEA Group } \\
\mathbf{N}=64 \\
22(34.38) \\
42(65.63)\end{array}$} & \multirow{2}{*}{$\begin{array}{l}\text { PT Group } \\
\mathbf{N}=64 \\
22(34.38) \\
42(65.63)\end{array}$} & \multirow{2}{*}{$\begin{array}{l}p \text {-value } \\
0.9999\end{array}$} \\
\hline Gender & $\begin{array}{l}\text { Male } \\
\text { Female }\end{array}$ & & & \\
\hline \multicolumn{2}{|l|}{ Age (year) } & $45.56[1.82]$ & $45.53[1.85]$ & 0.9904 \\
\hline \multicolumn{2}{|c|}{ Height (cm) } & $162.5[1.01]$ & $163.8[1.13]$ & 0.4001 \\
\hline \multicolumn{2}{|l|}{ Weight (kg) } & $65.35[1.88]$ & $63.69[1.29]$ & 0.4699 \\
\hline \multicolumn{2}{|c|}{ BMI $\left(\mathrm{kg} / \mathrm{m}^{2}\right)$} & $24.57[0.54]$ & $23.67[0.35]$ & 0.1622 \\
\hline \multicolumn{2}{|c|}{ Education (year) } & $14.59[0.4 \mid]$ & I5.05 [0.39] & 0.4214 \\
\hline Exercise & $\begin{array}{l}\text { Yes } \\
\text { No }\end{array}$ & $\begin{array}{l}39(60.94) \\
25(39.06)\end{array}$ & $\begin{array}{l}40(62.50) \\
24(37.50)\end{array}$ & 0.9999 \\
\hline Job & $\begin{array}{l}\text { Yes } \\
\text { No }\end{array}$ & $\begin{array}{l}43(67.19) \\
21(32.81)\end{array}$ & $\begin{array}{l}41(64.06) \\
23(35.94)\end{array}$ & 0.8525 \\
\hline Smoking & $\begin{array}{l}\text { Yes } \\
\text { No }\end{array}$ & $\begin{array}{l}9(14.06) \\
55(85.94)\end{array}$ & $\begin{array}{l}8(12.50) \\
56(87.50)\end{array}$ & 0.9999 \\
\hline Drinking & $\begin{array}{l}\text { Yes } \\
\text { No }\end{array}$ & $\begin{array}{l}31(48.44) \\
33(5 I .56)\end{array}$ & $\begin{array}{l}20(31.25) \\
44(68.75)\end{array}$ & 0.0705 \\
\hline $\begin{array}{l}\text { Treatment } \\
\text { history }\end{array}$ & $\begin{array}{l}\text { Yes } \\
\text { No }\end{array}$ & $\begin{array}{l}52(81.25) \\
12(18.75)\end{array}$ & $\begin{array}{l}43(67.19) \\
21(32.8 I)\end{array}$ & 0.1052 \\
\hline \multicolumn{2}{|c|}{$\begin{array}{l}\text { Symptom duration } \\
\text { (month) }\end{array}$} & $63.23[8.09]$ & $59.84[8.44]$ & 0.7723 \\
\hline
\end{tabular}

Note: Data are presented as $\mathrm{n}(\%)$ or mean [SD].

Abbreviations: BMI, body mass index; PT, physical therapy; TEA, thread embedding acupuncture.

subjects who dropped out of the study included 8 cases who withdrew consent for personal reasons, and 4 cases discontinued due to severe adverse events (SAEs). In the demographic characteristics and outcome variables at baseline, no significant differences were found between the two groups (Table 1).

In NPDS, the TEA group showed significant improvement compared to the PT group at all assessment points. The mean of NPDS scores at $9 \mathrm{~W}$ reduced from the baseline value of 24.48 points for the TEA group, while 6.76 points for the PT group. The adjusted mean difference of NPDS scores at $9 \mathrm{~W}$ between the two groups was -15.74 (95\% confidence interval [CI]: ( -22.05 to 9.44$)$ ), and the TEA group showed significant improved NPDS scores, compared to the PT group $(p<0.0001)$ (Table 2). The adjusted mean difference of NPDS scores at $5 \mathrm{~W}$ between the two groups was -15.30 (95\% CI: $(-21.03$ to -9.57$)$; $p<0.0001)$, and at $13 \mathrm{~W}$ was -14.93 (95\% CI: $(-21.20$ to 8.67); $p<0.0001)$. In the RM-ANOVA, the NPDS scores were analyzed using a Greenhouse-Geisser epsilon, since they did not meet the assumptions of the sphericity test, and showed a significant group $\times$ time interaction effect $(p<0.0001)$ (Figure 3).

In BDI-II, the TEA group showed significant improvement at $5 \mathrm{~W}(p=0.0211), 9 \mathrm{~W}(p=0.0261)$, and $13 \mathrm{~W}(p=$ 0.0108), compared to the PT group; and in BAI, the TEA group also showed significant improvement at $5 \mathrm{~W}(p=$ $0.0139)$ and $9 \mathrm{~W}(p=0.0127)$, compared with the PT group. In EQ-5D Index, the TEA group showed significant improvement at $13 \mathrm{~W}(p=0.0143)$, compared with the $\mathrm{PT}$ group; and in EQ-5D VAS, the TEA group showed significant improvement at $5 \mathrm{~W}(p=0.0003), 9 \mathrm{~W}(p=$ $0.0166)$, and $13 \mathrm{~W}(p=0.0330)$, compared with the PT group. However, in cervical spinal angle, no significant differences were found between the two groups (Table 2). Significant group $\times$ time interaction effects were found for the BDI-II $(p=0.0181)$, EQ-5D VAS $(p=0.0008)$ (Figure 3)

The proportions of patients with decreased NPDS scores of more than 11.5 points (minimal CID) were significantly higher in the TEA group than in the PT group at $5 \mathrm{~W}(p=0.0004), 9 \mathrm{~W}(p<0.0001)$, and $13 \mathrm{~W}(p=0.0003)$. In the moderate CID (decreased NPDS scores of more than 30\%) and substantial improvement (decreased NPDS scores of more than 50\%), significant superiority was also observed at all evaluation points in the TEA group (Figure 4). In PGIC, a significant larger number of patients in the TEA group than in the PT group reported as: "minimally improved", "much improved", or "very much improved" at 9W and 13W ( $p<0.0001)$ (Figure 5).

Among 1082 visitations, 22 mild AEs and 4 SAEs were reported. Among 22 mild AEs, 14 AEs were related to the TEA, 2 AEs were related to the PT, and 6 AEs were not associated with the intervention. Among 14 AEs related to the TEA, AEs from (2 to 7) days were 9, and AEs for more than 7 days were 5. Bruise was the most common discomfort, whereas others were stiffness in the neck region, pain, edema, pricking, and irritation caused by the thread. Among the 4 SAEs, 1 SAE was related to the TEA, while the other 3 SAEs were not related to the intervention. The SAE related to the TEA was iatrogenic pneumothorax (Table 3). Rescue medication was administered to $14(21.9 \%)$ subjects in the TEA group and 10 $(15.6 \%)$ in the PT group, with no statistically significant differences between the two groups.

\section{Discussion}

TEA treatment led to significant improvements in NPDS scores, compared to PT. The difference between the two 
Table 2 Observed Outcomes and Adjusted Group Differences

\begin{tabular}{|c|c|c|c|c|c|}
\hline Variables & Week & TEA Group & PT Group & Adjusted Mean Difference $^{a}(95 \% \mathrm{Cl})$ & p-value \\
\hline \multirow[t]{4}{*}{ NPDS } & Baseline & $55.29(1.84)$ & $51.57(1.89)$ & & 0.1622 \\
\hline & & $33.57(2.17)$ & $47.34(2.21)$ & $-15.30(-21.03,-9.57)$ & $<0.000 I^{*}$ \\
\hline & 9 & $30.81(2.43)$ & $44.81(2.50)$ & $-15.74(-22.05,9.44)$ & $<0.000 I^{*}$ \\
\hline & 13 & $32.56(2.42)$ & $45.73(2.44)$ & $-14.93(-21.20,-8.67)$ & $<0.000$ I* \\
\hline \multirow[t]{4}{*}{ BDI-II } & Baseline & I3.6I (0.94) & $12.20(1.03)$ & & 0.3155 \\
\hline & 5 & $11.40(1.04)$ & $12.64(1.28)$ & $-2.40(-4.44,-0.36)$ & $0.0211 *$ \\
\hline & 9 & $11.02(1.08)$ & $12.53(1.22)$ & $-2.54(-4.77,-0.30)$ & $0.0261^{*}$ \\
\hline & 13 & $10.21(1.05)$ & $12.56(1.20)$ & $-3.21(-5.67,-0.74)$ & $0.0108^{*}$ \\
\hline \multirow[t]{4}{*}{ BAI } & Baseline & $10.61(1.22)$ & $10.17(1.07)$ & & 0.7879 \\
\hline & & $6.57(0.84)$ & $9.49(1.23)$ & $-3.12(-5.61,-0.64)$ & $0.0139 *$ \\
\hline & 9 & $6.32(0.75)$ & 8.71 (0.97) & $-2.55(-4.56,-0.55)$ & $0.0127^{*}$ \\
\hline & 13 & $6.65(0.78)$ & $8.22(0.97)$ & $-1.7 \mid(-3.82,0.4 I)$ & 0.1133 \\
\hline \multirow[t]{4}{*}{ EQ-5D index } & Baseline & $0.864(0.011)$ & $0.874(0.011)$ & & 0.5111 \\
\hline & 5 & $0.883(0.010)$ & $0.853(0.020)$ & $0.035(-0.003,0.073)$ & 0.0700 \\
\hline & 9 & $0.873(0.018)$ & $0.842(0.020)$ & $0.038(-0.011,0.086)$ & 0.1306 \\
\hline & 13 & $0.887(0.011)$ & $0.839(0.021)$ & $0.052(0.010,0.094)$ & $0.0143 *$ \\
\hline \multirow[t]{4}{*}{ EQ-5D VAS } & Baseline & $59.22(2.44)$ & $62.19(2.04)$ & & 0.3521 \\
\hline & 5 & 70.55 (1.97) & $61.55(2.21)$ & $9.85(4.48,15.23)$ & $0.0003^{*}$ \\
\hline & 9 & $67.32(2.50)$ & $60.97(2.53)$ & $7.86(1.44, \mid 14.27)$ & $0.0166^{*}$ \\
\hline & 13 & $68.53(2.46)$ & $63.38(2.37)$ & $6.48(0.53,12.43)$ & $0.0330 *$ \\
\hline \multirow[t]{2}{*}{ Cervical spinal angle } & Baseline & $15.94(1.11)$ & $15.56(1.39)$ & & 0.8327 \\
\hline & 9 & $16.22(1.29)$ & $15.35(1.57)$ & $0.65(-2.58,3.88)$ & 0.6921 \\
\hline
\end{tabular}

Notes: ${ }^{*} p<0.05$. Data are presented as mean (SD). ${ }^{a}$ Calculated from ANCOVA with baseline score as a covariate.

Abbreviations: ANCOVA, analysis of covariance; BAI, Beck Anxiety Inventory; BDI-II, Beck Depression Inventory II; Cl, confidence interval; EQ-5D, EuroQol FiveDimension; NPDS, Neck Pain and Disability Scale; PT, physical therapy; TEA, thread embedding acupuncture; VAS, visual analogue scale.

groups in NPDS scores tended to increase from $5 \mathrm{~W}$ to $9 \mathrm{~W}$, and was also significant even up to $13 \mathrm{~W}$. This is a result similar to the previous studies, which showed improvement in the NPDS scores as it approached $9 \mathrm{~W} .{ }^{16}$ Considering that chronic pain tends to be stubborn, it is noteworthy that the difference in NPDS scores between the two groups remained at around 15 points at all followup assessments ( 1 week, 5 weeks, and 9 weeks after the last intervention). Also, significant improvements in BDIII, BAI, and EQ-5D were observed in the TEA group, compared with the PT group. These results suggest that TEA may be effective in improving psychological distress and quality of life of CNP patients, and is consistent with the finding of a previous study that neck pain is significantly correlated with anxiety and depression. ${ }^{46}$ The proportion of patients with decreased NPDS scores of more than minimal CID, $30 \%$, and 50\% was significantly higher in the TEA group, compared with the PT group. Therefore, TEA treatment is not only statistically more effective in the treatment of CNP patients than the PT, but also clinically meaningful. Additionally, the percentage of subjects who answered "improved" in TEA group was $96.5 \%$ at $9 \mathrm{~W}$ and $98.2 \%$ at $13 \mathrm{~W}$. These percentages were significantly higher than those in the PT group. In addition, there were significant group $\times$ time interaction effects in NPDS, BDI-II, and EQ-5D. However, in cervical spinal angle, there was no significant difference between the two groups before and after the intervention. The result was consistent with other studies that failed to show significant correlation between neck pain and cervical angle because of multidimensional factors, such as imbalance of muscle tone, poor posture, lifestyle, and occupation affecting the cervical angle. $^{39,44}$

Analysis of AEs related to intervention showed that most AEs were mild, and disappeared within a few days. SAE definitely related to TEA was iatrogenic pneumothorax. This subject was treated with inpatient and outpatient treatments, and under the supervision of the researchers. Radiological imaging tests and examination by a research physician on the 16th day after the 

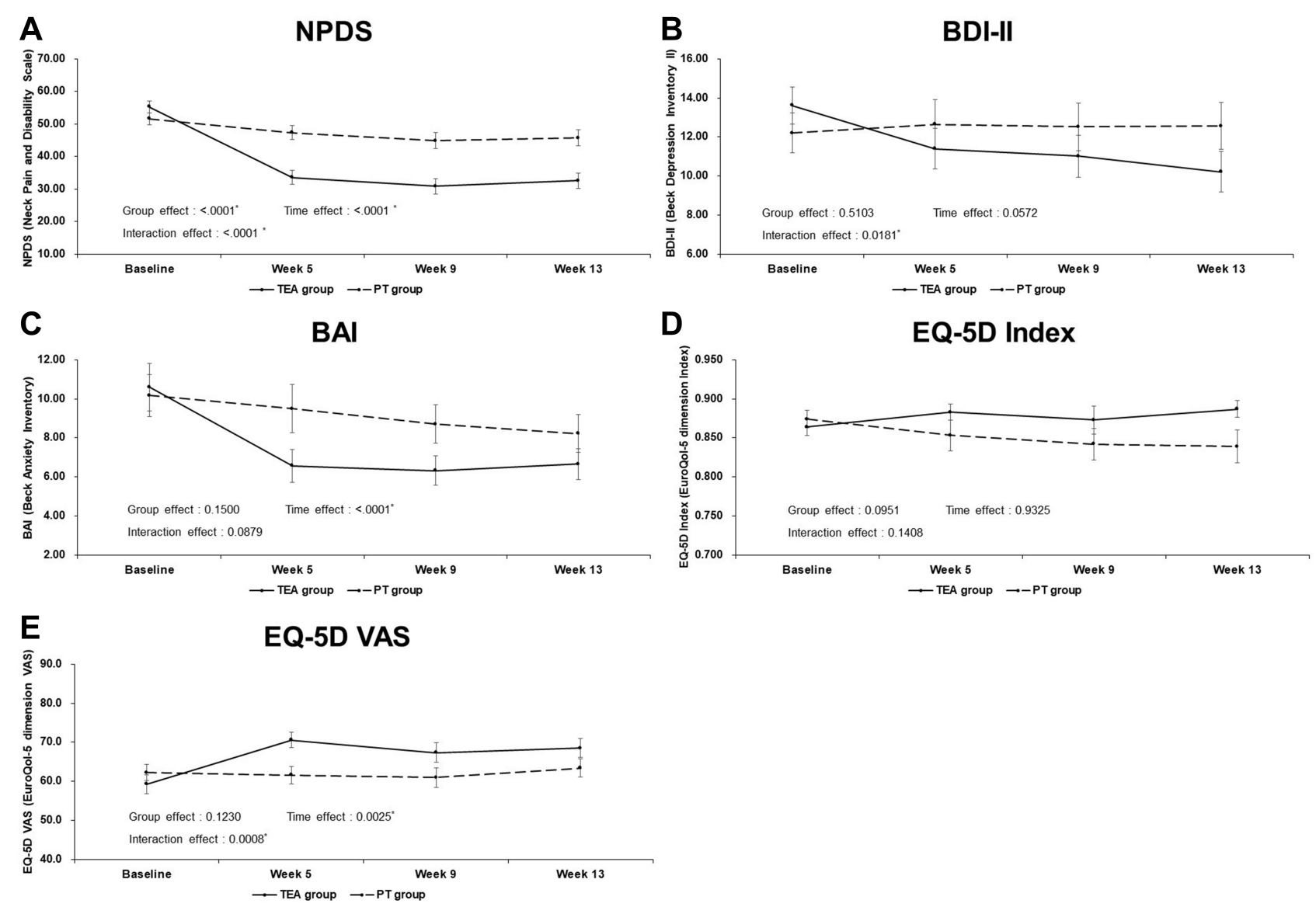

Figure 3 Change over time on NPDS, BDI-II, BAI, EQ-5D Index, and EQ-5D VAS. The group $x$ time interaction effect, which determined from analyses using a repeated measures analysis of variance, was significant in NPDS (A), BDI-II (B), and EQ-5D VAS (E), but not in BAI (C) and EQ-5D index (D).

Note: $*_{p}<0.05$.

Abbreviations: BAI, Beck Anxiety Inventory; BDI-II, Beck Depression Inventory II; EQ-5D, EuroQol Five-Dimensions; NPDS, Neck Pain and Disability Scale; PT, physical therapy; TEA, thread embedding acupuncture; VAS, visual analogue scale.

occurrence of pneumothorax confirmed that all related symptoms had disappeared. The practitioners were trained to prevent possible side effects during the procedure, including pneumothorax. However, it was difficult to completely eliminate the possibility of procedural errors. The practitioners were retrained to prevent further AEs. However, the pneumothorax was caused by the practitioner's mistake, and as mentioned above, other side effects caused by the TEA treatment were mostly mild.

The results of this study showed that TEA treatment was more effective than PT treatments in improving pain, psychological distress, and the quality of life of CNP patients. In addition, we obtained the results of longer-term observation after TEA treatment compared to previous studies, ${ }^{15,16}$ and found high satisfaction with TEA treatment, significant clinical relevance of TEA treatment, and possible adverse events after TEA treatment. In this study, the treatment frequency of the two interventions was different. TEA was administered once weekly, whereas PT twice weekly because this study focused on verifying external validity based on actual clinical practice as well as treatment frequency of TEA and PT in previous studies. ${ }^{15,16,57}$ Nevertheless, it was noteworthy that fewer sessions of TEA yielded better outcomes than PT. The therapeutic mechanism of TEA has yet to be clearly established; however, it is suggested that longlasting stimulation of afferent fibers by embedded PDO threads contributes to pain relief. ${ }^{58}$

However, this study has some limitations. First, due to the obvious differences in intervention between the two groups, practitioners and subjects could not be blinded. This could have led to non-specific effects that were not related to interventions, such as treatment expectations or placebo, and the possibility that these non-specific effects had more favorable effects on the TEA group was not 


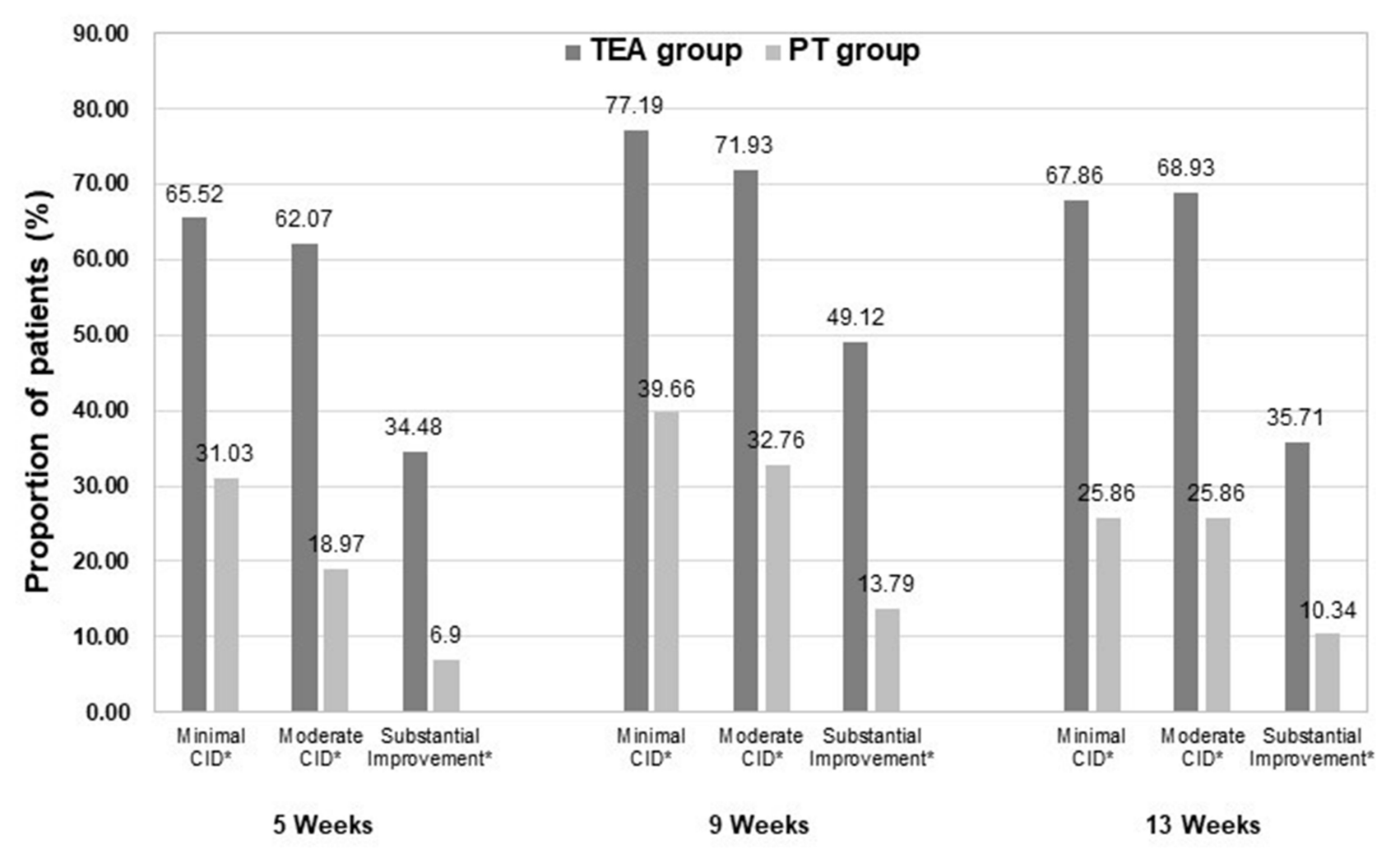

Figure 4 Clinical relevance.

Note: $*_{p}<0.05$.

Abbreviations: CID, clinically important difference; PT, physical therapy; TEA, thread embedding acupuncture.

PGIC

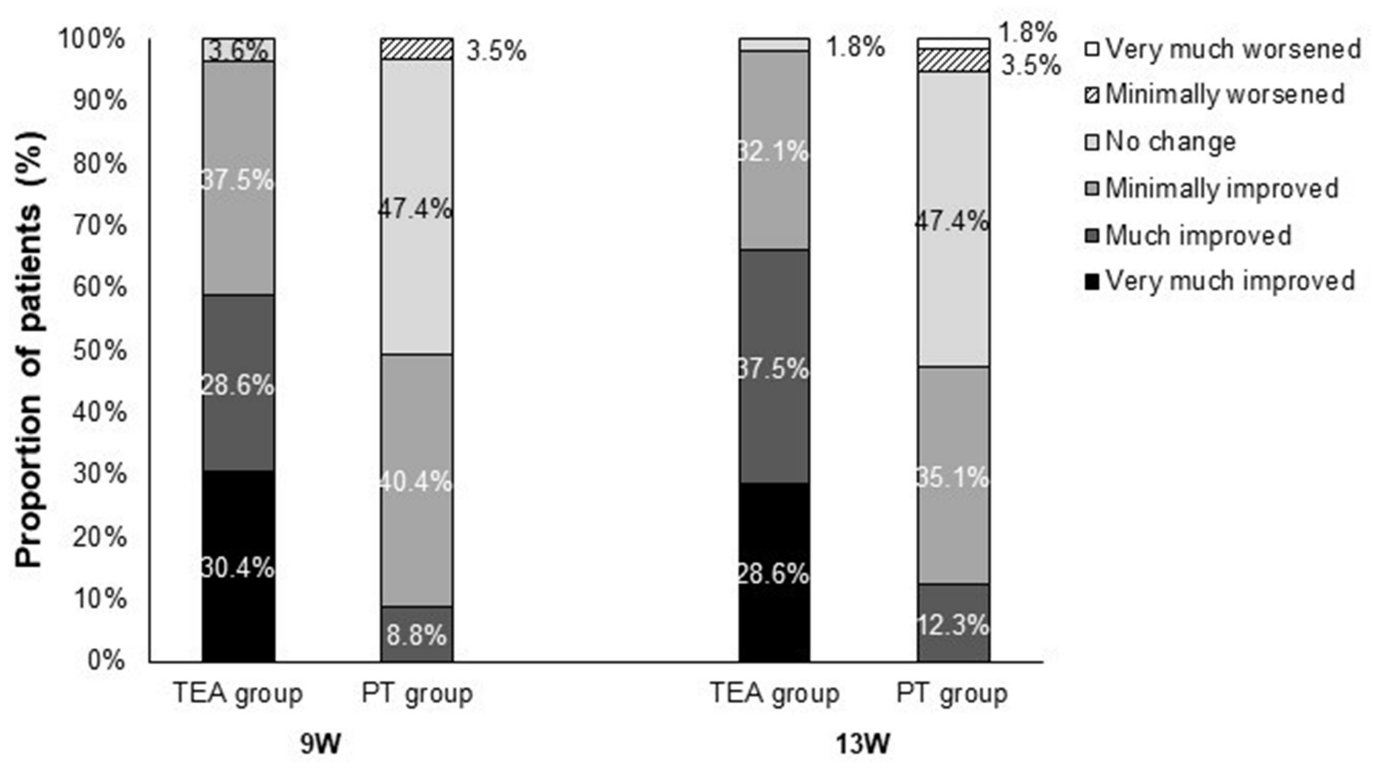

Figure 5 Patient global impression of change.

Abbreviations: PGIC, patient global impression of change; PT, physical therapy; TEA, thread embedding acupuncture. 
Table 3 Summary of Adverse Events

\begin{tabular}{|c|c|c|c|}
\hline Variables & $\begin{array}{l}\text { TEA } \\
\text { Group }\end{array}$ & $\begin{array}{l}\text { PT } \\
\text { Group }\end{array}$ & Total \\
\hline $\begin{array}{l}\text { Total visits of each intervention, } \mathrm{n}(\%) \\
\text { Total adverse events, } \mathrm{n}(\%)\end{array}$ & $\begin{array}{l}414 \\
(100.00) \\
21(5.07)\end{array}$ & $\begin{array}{l}668 \\
(100.00) \\
5(0.75)\end{array}$ & $\begin{array}{l}1082 \\
(100.00) \\
26(2.40)\end{array}$ \\
\hline Mild adverse events & & & \\
\hline $\begin{array}{l}\text { Definitely related to TEA } \\
\text { (2 to } 7 \text { ) days } \\
\text { Stiffness, } \mathrm{n} \text { (mean duration in days) } \\
\text { Bruise, } \mathrm{n} \text { (mean duration in days) } \\
\text { Pain, } \mathrm{n} \text { (mean duration in days) } \\
\text { Edema, } \mathrm{n} \text { (mean duration in days) } \\
\text { Pricking, } \mathrm{n} \text { (mean duration in days) } \\
\text { More than } 7 \text { days } \\
\text { Bruise, } \mathrm{n} \text { (mean duration in days) } \\
\text { Irritation, } \mathrm{n} \text { (mean duration in days) } \\
\text { Definitely related to PT } \\
\text { Stiffness, } \mathrm{n} \text { (mean duration in days) } \\
\text { Pain, } \mathrm{n} \text { (mean duration in days) } \\
\text { Not related to intervention, } \mathrm{n}\end{array}$ & $\begin{array}{l}3(4.00) \\
2(5.00) \\
2(3.50) \\
\text { I }(2.00) \\
\text { I }(3.00) \\
4(12.25) \\
\text { I }(7.00)\end{array}$ & $\begin{array}{l}I(3.00) \\
I(12.00) \\
2\end{array}$ & \\
\hline \multicolumn{4}{|l|}{ Severe adverse events } \\
\hline $\begin{array}{l}\text { Definitely related to TEA } \\
\text { Pneumothorax, } \mathrm{n} \text { (mean duration } \\
\text { in days) } \\
\text { Not related to intervention, } \mathrm{n}\end{array}$ & $\begin{array}{l}1(16.00) \\
2\end{array}$ & I & \\
\hline
\end{tabular}

Abbreviations: PT, physical therapy; TEA, thread embedding acupuncture.

completely excluded. Also, although TEA treatments were found to be significantly more effective than PT treatments, it was difficult to conclude that TEA treatments completely relieved pain, as patients in the TEA group also maintained NPDS scores between about 30 to 35 . The follow-up period of 13 weeks was also not enough to measure the long-term effects of TEA. In addition, pneumothorax, which is an SAE, occurred due to the practitioner's errors.

Despite these limitations, this was the first clinical trial to compare the effectiveness and safety of TEA with PT treatment in the treatment of patients with CNP, which confirmed the superiority of TEA treatment to PT. In addition, this study provides a clinically beneficial and objective outcome for comparing the effects of the two different interventions, and these results are expected to be used as data for research to verify the effectiveness of TEA treatments. In future research, we will intend to conduct long-term follow-up, multi-center clinical trials using TEA; and furthermore, to conduct studies that evaluate the effects of TEA and other complementary and alternative medicine treatments of patients with other musculoskeletal diseases, including CNP.

\section{Conclusion}

In the treatments of patients with CNP, TEA treatment showed significant improvement, compared to PT treatment. The improvements in the pain and dysfunction, psychological distress, quality of life, and satisfaction of patients in the TEA group were higher than those of patients in the PT group, and the side effects after TEA treatment were mostly mild. Therefore, TEA can be considered as a useful treatment option for patients with CNP.

\section{Abbreviations}

$\mathrm{AE}$, adverse event; ANCOVA, analysis of covariance; BAI, Beck Anxiety Inventory; BDI-II, Beck Depression Inventory II; BMI, body mass index; CI, confidence interval; CID, clinically important difference; CNP, non-specific chronic neck pain; CRF, case report form; EQ-5D-3L, EuroQol Five-Dimensions 3-Level version; FAS, full analysis set; ICT, interferential current therapy; NPDS, Neck Pain and Disability Scale; PDO, polydioxanone; PGIC, patient global impression of change; PP, per protocol; PT, physical therapy; RM-ANOVA, repeated measures analysis of variance; SAE, severe adverse event; TEA, thread embedding acupuncture; VAS, visual analogue scale.

\section{Data Sharing Statement}

The datasets generated during this study are available from Eunseok Kim, the corresponding author, on reasonable request.

\section{Ethics Approval and Consent to Participate}

This clinical trial was approved by the Institutional Review Board (DJDSKH-19-BM-04), registered with the Clinical Research Information Service (KCT0003720), and conducted in accordance with the Declaration of Helsinki. All applicants were provided a written explanation and an informed consent form, and received a full explanation of this trial.

\section{Author Contributions}

Jae Ik Kim and Eunseok Kim drafted the manuscript, and conducted TEA treatment. Young Il Kim and Chang-Hyun Han contributed to the conceptualization and supervision of this study. All authors made substantial contributions to conception, study design, and data analysis; took part in 
revising the article critically for important intellectual content; agreed to submit to the current journal; gave final approval of the version to be published; and agree to be accountable for all aspects of the work.

\section{Funding}

This study was financially supported by grants from project KSN2021210 of the Korea Institute of Oriental Medicine (KIOM), Republic of Korea. The funding sources had no role in the experimental design, procedures, writing, and decision to submit the work for publication.

\section{Disclosure}

The authors have no conflicts of interest to declare.

\section{References}

1. Binder AI. Cervical spondylosis and neck pain. $B M J$. 2007;334:527-531. doi:10.1136/bmj.39127.608299.80

2. Brattberg G, Thorslund M, Wikman A. The prevalence of pain in a general population. The results of a postal survey in a county of Sweden. Pain. 1989;37:215-222. doi:10.1016/0304-3959(89)90133-4

3. Hogg-Johnson S, van der Velde G, Carroll LJ, et al. The burden and determinants of neck pain in the general population: results of the Bone and Joint Decade 2000-2010 Task Force on Neck Pain and Its Associated Disorders. J Manipulative Physiol Ther. 2009;32:S46-60. doi:10.1016/j.jmpt.2008.11.010

4. Cote P, van der Velde G, Cassidy JD, et al. The burden and determinants of neck pain in workers: results of the Bone and Joint Decade 2010 Task Force on Neck Pain and Its Associated Disorders. $J$ Manipulative Physiol Ther. 2009;32:S70-86. doi:10.1016/j. jmpt.2008.11.012

5. Hoy D, March L, Woolf A, et al. The global burden of neck pain: estimates from the global burden of disease 2010 study. Ann Rheum Dis. 2014;73:1309-1315. doi:10.1136/annrheumdis-2013-204431

6. Trinh K, Graham N, Irnich D, Cameron ID, Forget M. Acupuncture for neck disorders. Cochrane Database Syst Rev. 2016;5:CD004870.

7. Witt CM, Jena S, Brinkhaus B, Liecker B, Wegscheider K, Willich SN. Acupuncture for patients with chronic neck pain. Pain. 2006;125(1):98-106. doi:10.1016/j.pain.2006.05.013

8. Salter GC, Roman M, Bland MJ, MacPherson H. Acupuncture for chronic neck pain: a pilot for a randomised controlled trial. $B M C$ Musculoskelet Disord. 2006;7(1):99. doi:10.1186/1471-2474-7-99

9. Kim YJ, Kim HN, Shin MS, Choi BT. Thread embedding acupuncture inhibits ultraviolet B irradiation-induced skin photoaging in hairless mice. Evid Based Complement Alternat Med. 2015;2015:1-9.

10. Acupuncture and Moxibustion Textbook Compilation Committee. The Acupuncture \& Moxibustion Medicine. Seoul: Jipmoondang; 2016.

11. Qin W, Zhao K, Yang H. Effect of acupoint catgut embedding therapy combined with Chinese medicine for nourishing the kidneys and promoting blood circulation and improving blood glucose and lipid levels as well as the pregnancy rate in obese PCOS patients with infertility. Exp Ther Med. 2016;12(5):2909-2914. doi:10.3892/etm. 2016.3715

12. Garcia-Vivas JM, Galaviz-Hernandez C, Fernandez-Retana J, et al. Transcriptomic profiling of adipose tissue in obese women in response to acupuncture catgut embedding therapy with moxibustion. $J$ Altern Complement Med. 2016;22(8):658-668. doi:10.1089/acm.2015.0200
13. Chen GZ, Xu YX, Zhang JW, Liu SH, Guo ZY. Effect of acupoint catgut-embedding on the quality of life, reproductive endocrine and bone metabolism of postmenopausal women. Chin J Integr Med. 2010;16(6):498-503. doi:10.1007/s11655-010-0574-1

14. Karabulut R, Sonmez K, Turkyilmaz Z, Bagbanci B, Basaklar AC, Kale N. An in vitro and in vivo evaluation of tensile strength and durability of seven suture materials in various $\mathrm{pH}$ and different conditions: an experimental study in rats. Indian J Surg. 2010;72 (5):386-390. doi:10.1007/s12262-010-0158-5

15. Kim E, Kim HS, Jung SY, Han CH, Kim YI. Efficacy and safety of polydioxanone thread embedded at specific acupoints for non-specific chronic neck pain: a study protocol for a randomized, subject-assessor-blinded, sham-controlled pilot trial. Trials. 2018;19 (1):672. doi:10.1186/s13063-018-3058-9

16. Kim E, Kim YS, Kim YI, et al. Effectiveness and safety of polydioxanone thread-embedding acupuncture as an adjunctive therapy for patients with chronic nonspecific neck pain: a randomized controlled trial. J Altern Complement Med. 2019;25(4):417-426. doi:10.1089/acm.2018.0228

17. Facci LM, Nowotny JP, Tormem F, Trevisani VFM. Effects of transcutaneous electrical nerve stimulation (TENS) and interferential currents (IFC) in patients with nonspecific chronic low back pain: randomized clinical trial. Sao Paulo Med J. 2011;129(4):206-216. doi:10.1590/S1516-31802011000400003

18. Yesil H, Hepguler S, Dundar U, Taravati S, Isleten B. Does the use of electrotherapies increase the effectiveness of neck stabilization exercises for improving pain, disability, mood, and quality of life in chronic neck pain?: a randomized, controlled, single-blind study. Spine. 2018;43(20):E1174-1183. doi:10.1097/BRS.000000 0000002663

19. Acedo AA, Antunes ACL, Dos Santos AB, et al. Upper trapezius relaxation induced by TENS and interferential current in computer users with chronic nonspecific neck discomfort: an electromyographic analysis. J Back Musculoskelet Rehabil. 2015;28(1):19-24. doi:10.3233/BMR-140482

20. Fuentes JP, Armijo Olivo SA, Magee DJ, Gross DP. Effectiveness of interferential current therapy in the management of musculoskeletal pain: a systematic review and meta-analysis. Phys Ther. 2010;90 (9):1219-1238. doi:10.2522/ptj.20090335

21. Suh CY, Lee YJ, Kim MR, et al. A web-based survey for assessment of Korean medical treatment clinical practice patterns for neck pain and cervical intervertebral disc displacement. J Acupunct Res. 2016;33(4):65-72. doi:10.13045/acupunct.2016055

22. White P, Lewith G, Prescott P, Conway J. Acupuncture versus Placebo for the treatment of chronic mechanical neck pain. Ann Intern Med. 2004;141(12):911. doi:10.7326/0003-4819-141-12200412210-00007

23. Jeon GS, Choi ES, Lee HY. Gender-related difference in the utilization of health care services by Korean adults. J Korean Acad Public Health Nurs. 2010;24(2):182-196.

24. Jung H, Park H, Lee S. Nationwide study on the characteristics of patients visiting and using Korean medical facilities by sex. $J$ Korean Med. 2014;35(1):75-87. doi:10.13048/jkm.14008

25. Seo YJ, Kang SH, Kim YH, Choi DB, Shin HK. Customers' utilization and satisfaction in oriental medical clinics. $J$ Korean Med. 2010;31(2):124-136.

26. Park JE, Kwon SM. Determinants of the utilization of oriental medical services by the elderly. $J$ Korean Med. 2011;32(1):97-108.

27. Yoo WK, Ryu KA. Utilization patterns and determinants of oriental medical services: focused on the residents of Taegu City. J Soc Prev Korean Med. 2000;4(2):1-24.

28. Jorritsma W, Dijkstra PU, de Vries GE, Geertzen JHB, Reneman MF. Detecting relevant changes and responsiveness of neck pain and disability scale and neck disability index. Eur Spine J. 2012;21 (12):2550-2557. doi:10.1007/s00586-012-2407-8 
29. Kim JI, Kim YI, Kim E, et al. Effectiveness and safety of polydioxanone thread embedding acupuncture compared to physical therapy in the treatment of patients with non-specific chronic neck pain: study protocol for an assessor-blinded, randomized, controlled, clinical trial. Medicine. 2019;98(32):e16768. doi:10.1097/MD.0000000000 016768

30. Kuorinka I, Jonsson B, Kilbom A, et al. Standardised nordic questionnaires for the analysis of musculoskeletal symptoms. Appl Ergon. 1987;18(3):233-237. doi:10.1016/0003-6870(87)90010-X

31. Aker PD, Gross AR, Goldsmith CH, Peloso P. Conservative management of mechanical neck pain: systematic overview and meta-analysis. BMJ. 1996;313(7068):1291-1296. doi:10.1136/bmj.31 3.7068.1291

32. Waling K, Jaörvholm B, Sundelin G. Effects of training on female trapezius myalgia: an intervention study with a 3-year follow-up period. Spine. 2002;27(8):789-796. doi:10.1097/00007632-20020415 0-00002

33. Hurwitz EL, Aker PD, Adams AH, Meeker WC, Shekelle PG. Manipulation and mobilization of the cervical spine: a systematic review of the literature. Spine. 1996;21(15):1746-1760. doi:10.10 97/00007632-199608010-00007

34. The Society of Korean Medicine Rehabilitation. Oriental Rehabilitation Medicine. Seoul: Koonja; 2011.

35. Wheeler AH, Goolkasian P, Baird AC, Darden BV. Development of the neck pain and disability scale. Item analysis, face, and criterion-related validity. Spine. 1999;24(13):1290-1294. doi:10.10 97/00007632-199907010-00004

36. Lee JS, Suh KT, Kim JI, Lee HS, Goh TS. Validation of the Korean version of the neck pain and disability scale. Asian Spine J. 2013;7 (3):178-183. doi:10.4184/asj.2013.7.3.178

37. Jaeschke R, Singer J, Guyatt GH. Measurement of health status. Ascertaining the minimal clinically important difference. Control Clin Trials. 1989;10(4):407-415. doi:10.1016/0197-2456(89)90005-6

38. Dworkin RH, Turk DC, Wyrwich KW, et al. Interpreting the clinical importance of treatment outcomes in chronic pain clinical trials: IMMPACT recommendations. J Pain. 2008;9(2):105-121. doi:10.10 16/j.jpain.2007.09.005

39. Gore DR, Sepic SB, Gardner GM. Roentgenographic findings of the cervical spine in asymptomatic people. Spine. 1986;11(6):521-524 doi:10.1097/00007632-198607000-00003

40. Harrison DE, Harrison DD, Cailliet R, Troyanovich SJ, Janik TJ, Holland B. Cobb method or Harrison posterior tangent method: which to choose for lateral cervical radiographic analysis. Spine. 2000;25(16):2072-2078. doi:10.1097/00007632-200008150-00011

41. Harrison DD, Harrison DE, Janik TJ, et al. Modeling of the sagittal cervical spine as a method to discriminate hypolordosis: results of elliptical and circular modeling in 72 asymptomatic subjects, 52 acute neck pain subjects, and 70 chronic neck pain subjects. Spine. 2004;29 (22):2485-2492. doi:10.1097/01.brs.0000144449.90741.7c

42. Askin A, Bayram KB, Demirdal US, et al. The evaluation of cervical spinal angle in patients with acute and chronic neck pain. Turk $J$ Med Sci. 2017;47(3):806-811. doi:10.3906/sag-1601-179
43. McAviney J, Schulz D, Bock R, Harrison DE, Holland B Determining the relationship between cervical lordosis and neck complaints. J Manipulative Physiol Ther. 2005;28(3):187-193. doi:10.1016/j.jmpt.2005.02.015

44. Grob D, Frauenfelder H, Mannion AF. The association between cervical spine curvature and neck pain. Eur Spine J. 2007;16 (5):669-678. doi:10.1007/s00586-006-0254-1

45. Helliwell PS, Evans PF, Wright V. The straight cervical spine: does it indicate muscle spasm? J Bone Joint Surg. 1994;76(1):103-106. doi:10.1302/0301-620X.76B1.8300650

46. Blozik E, Laptinskaya D, Herrmann-Lingen C, et al. Depression and anxiety as major determinants of neck pain: a cross-sectional study in general practice. BMC Musculoskelet Disord. 2009;10(1):13. doi:10.1186/1471-2474-10-13

47. Beck AT, Steer RA, Brown GK. Manual for the BDI-II. San Antonio TX: The Psychological Corporation; 1996.

48. Kim JH, Lee EH, Hwang ST, Hong SH. Korean-Beck Depression Inventory II. Daegu: Korea Psychol; 2015.

49. Beck AT, Epstein N, Brown G, Steer RA. An inventory for measuring clinical anxiety: psychometric properties. J Consult Clin Psychol. 1988;56(6):893-897. doi:10.1037/0022-006X.56.6.893

50. Kim JH, Lee EH, Hwang ST, Hong SH. Korean-Beck Anxiety Inventory. Daegu: Korea Psychol; 2015.

51. Seong SS, Choi CB, Sung YK, et al. Health-related quality of life using EQ-5D in Koreans. J Korean Rheum Assoc. 2004;11 (3):254-262.

52. Kim MH, Cho YS, Uhm WS, Kim S, Bae SC. Cross-cultural adaptation and validation of the Korean version of the EQ-5D in patients with rheumatic diseases. Qual Life Res. 2005;14(5):1401-1406. doi:10.1007/s11136-004-5681-z

53. Farrar JT, Young JP Jr, LaMoreaux L, Werth JL, Poole MR. Clinical importance of changes in chronic pain intensity measured on an 11-point numerical pain rating scale. Pain. 2001;94(2):149-158. doi:10.1016/S0304-3959(01)00349-9

54. Tantikul C, Dhana N, Jongjarearnprasert K, Visitsunthorn N, Vichyanond P, Jirapongsananuruk O. The utility of the World Health Organization-The Uppsala Monitoring Centre (WHO-UMC) system for the assessment of adverse drug reactions in hospitalized children. Asian Pac J Allergy Immunol. 2008;26(2-3):77-82.

55. Spilker B. Interpretation of adverse reactions. In: Spilker B, editors. Guide to Clinical Trials. New York: Raven Press. 1991:565-587.

56. Cotchett MP, Munteanu SE, Landorf KB. Effectiveness of trigger point dry needling for plantar heel pain: a randomized controlled trial. Phys Ther. 2014;94(8):1083-1094. doi:10.2522/ptj.20130255

57. Park SH Literature Review of Catgut-Embedding Therapy for Lumbar Disk Herniation [dissertation]. Wanju: Woosuk University; 2015. Korean.

58. Zhao ZQ. Neural mechanism underlying acupuncture analgesia. Prog Neurobiol. 2008;85(4):355-375. doi:10.1016/j.pneurobio.2008.05. 004
Journal of Pain Research

\section{Publish your work in this journal}

The Journal of Pain Research is an international, peer reviewed, open access, online journal that welcomes laboratory and clinical findings in the fields of pain research and the prevention and management of pain. Original research, reviews, symposium reports, hypothesis formation and commentaries are all considered for publication. The manuscript

Submit your manuscript here: https://www.dovepress.com/journal-of-pain-research-journal management system is completely online and includes a very quick and fair peer-review system, which is all easy to use. Visit http:// www.dovepress.com/testimonials.php to read real quotes from published authors. 